\title{
Retraction Note: Comparison of the effect of alendronate on lumbar bone mineral density and bone turnover in men and postmenopausal women with osteoporosis
}

\author{
Jun Iwamoto ${ }^{1} \cdot$ Tsuyoshi Takeda $^{1} \cdot$ Yoshihiro Sato $^{2} \cdot$ Mitsuyoshi Uzawa $^{3}$
}

Published online: 18 June 2021

(C) International League of Associations for Rheumatology (ILAR) 2021

\section{Retraction Note: Clin Rheumatol (2007) 26: 161-167}

https://doi.org/10.1007/s10067-006-0252-Z

The Editor-in-Chief has retracted this article [1]. After publication, serious concerns were raised with respect to the ethical oversight, methodology and authorship. Keiyu Orthopaedic Hospital has not been able to confirm that appropriate ethics approval was obtained. The Editor-in-Chief no longer has confidence in this article. Jun Iwamoto has not responded to correspondence from the Editor-in-Chief or publisher about this retraction. The Editor-in-Chief and the publisher were not able to obtain current email addresses for Tsuyoshi Takeda and Mitsuyoshi Uzawa. Yoshiro Sato is deceased.

\section{Reference}

1. Iwamoto J, Takeda T, Sato Y, Uzawa M (2007) Comparison of the effect of alendronate on lumbar bone mineral density and bone turnover in men and postmenopausal women with osteoporosis. Clin Rheumatol 26:161-167. https://doi.org/10.1007/ s10067-006-0252-z

Publisher's note Springer Nature remains neutral with regard to jurisdictional claims in published maps and institutional affiliations.

The online version of the original article can be found at https:// doi.org/10.1007/s10067-006-0252-z

Jun Iwamoto

jiwamoto@sc.itc.keio.ac.jp

1 Department of Sports Medicine, Keio University, School of Medicine, 35 Shinanomachi, Shinjuku-ku, Tokyo 160-8582, Japan

2 Department of Neurology, Mitate Hospital, Fukuoka, Japan

3 Department of Orthopaedic Surgery, Keiyu Orthopaedic Hospital, Gunma, Japan 